\title{
Etude de la prévalence de la brucellose bovine en zone forestière de la Côte d'Ivoire
}

\author{
E. Thys ${ }^{1 *}$ M.A. Yahaya ${ }^{2}$ K. Walravens ${ }^{3}$ C. Baudoux ${ }^{4}$ \\ I. Bagayoko ${ }^{4}$ D. Berkvens ${ }^{1}$ S. Geerts ${ }^{1}$
}

Mots-clés

Bovin - Brucella - Brucellose Morbidité - Technique immunologique - Zone périurbaine - Forêt - Côte d'Ivoire.

\section{Résumé}

Une enquête sérologique a été menée dans la zone forestière périurbaine d'Abidjan entre juillet et septembre 2004, afin d'évaluer la prévalence de la brucellose dans le cheptel laitier bovin. Trois cent quatre vingt et un (381) sérums ont été récoltés sur un total de 927 animaux, dont 506 provenaient des fermes laitières suivies par le Projet laitier Sud (fermes d'application et privées) et 421 des élevages traditionnels environnants. Quatre tests sérologiques ont été appliqués : le test au rose bengale, l'Elisa indirect, le test de fixation du complément et I'agglutination lente de Wright avec Edta (microméthode). Sur la base des analyses sérologiques et bayésienne, des séroprévalences de l'ordre de 3,6 et 4,3 p. 100 ont été trouvées respectivement dans les fermes laitières et dans les élevages traditionnels. II n’y a pas eu de différence significative entre les fermes laitières et les élevages traditionnels $(p<0,05)$.

\section{INTRODUCTION}

La brucellose est une maladie fortement pathogène causée par des bactéries du genre Brucella. Elle est considérée comme la zoonose la plus répandue dans le monde (15). Elle provoque de très importantes pertes économiques en élevage $(12,13,16,20)$ et représente un danger de santé publique non négligeable (17), surtout dans les régions du monde où le bétail est la source principale d'aliments et de revenus (8).

La brucellose est présente en Afrique subsaharienne où elle est considérée comme un problème majeur chez les ruminants (24). La prévalence montre une forte variabilité selon les pays et les régions

\footnotetext{
1. Institut de médecine tropicale, Anvers, B-2000 Belgique.

2. s/c Ets Houdou Younoussa, Niamey, Niger.

3. Centre d'étude et de recherches vétérinaires et agrochimiques d'Uccle, Bruxelles, B-1180 Belgique.

4. Projet laitier Sud, Abidjan, Côte d'Ivoire.

* Auteur pour la correspondance

Institut de médecine tropicale, département de santé animale,

Nationalestraat 155, 2000 Anvers, Belgique.

Tél. : +32 (0)32476392; fax : +32(0)324762 68

E-mail : ethys@itg.be
}

$(1,5,7,15)$. En Côte d'Ivoire, les foyers de brucellose bovine ont été principalement détectés au Nord, avec des taux d'infection de l'ordre de 12 à 14 p. 100 (3). Entre 1975 et 1977, un taux de positivité de 10,8 p. 100 sur l'ensemble du territoire avait été déterminé par le laboratoire de pathologie animale de Bingerville (20).

L'absence de données récentes sur la prévalence de la brucellose bovine en Côte d'Ivoire et plus particulièrement en zone méridionale forestière donne toute son importance à la présente étude. En effet, l'élevage laitier est en train de se développer dans la zone forestière tout autour de la capitale. Une ferme d'application a été installée près d'Abidjan, dans le cadre d'un projet belgo-ivoirien, le Projet laitier Sud (PLS), qui doit fournir des animaux de qualité supérieure à des éleveurs privés démarrant une ferme laitière et former ces derniers aux différentes techniques liées à ce type d'élevage. La consommation du lait frais devenant une réalité dans cette partie du pays, il s'est donc avéré utile de connaître la prévalence de la brucellose aussi bien chez les animaux de la ferme d'application, que parmi ceux distribués aux fermes laitières débutantes. Des troupeaux extensifs traditionnels gardés par des bergers Peuls coexistent dans la zone. Leur statut sérologique en matière de brucellose a été tout aussi important à déterminer compte tenu des risques de contamination possibles. 


\section{MATERIEL ET METHODES}

\section{Contexte et collecte des données}

La collecte des données a été effectuée entre juillet et septembre 2004 dans les districts d'Abidjan, de Bingerville, d'Azaguié et de Makouguié, situés respectivement à 2, 40 et $50 \mathrm{~km}$ d'Abidjan, dans le Sud forestier de la Côte d'Ivoire (figure 1). Elle a concerné le troupeau de la ferme d'application du projet PLS à Bingerville et les fermes laitières privées suivies par le projet, avec 244 animaux de plus d'un an échantillonnés aléatoirement sur un total de 506 $(48,2$ p. 100) dans dix fermes différentes. Aucune vaccination systématique n'était appliquée sur place sur les animaux qui provenaient en majorité de stations d'Etat et d'élevages privés situés au centre et au nord du pays (Séguéla, Korhogo, Bouaké). A titre de comparaison et afin d'obtenir une idée de la prévalence de la brucellose dans les élevages traditionnels, 137 animaux sur 421 (32,5 p. 100) ont été échantillonnés dans 12 troupeaux installés majoritairement à la périphérie des agglomérations, à l'exception d'un seul qui se trouvait en pleine forêt tout comme les fermes laitières.

Au total 381 sérums ont ainsi été collectés. Des informations complémentaires ont été recueillies sur le sexe de chaque animal prélevé, son âge, son origine ainsi que le nom de l'éleveur concerné. Les sérums recueillis ont été conservés au congélateur à $-20{ }^{\circ} \mathrm{C}$ jusqu'à leur arrivée au Centre d'étude et de recherches vétérinaires et agrochimiques (Cerva, Uccle, Belgique) pour les analyses sérologiques.

\section{Analyses sérologiques}

Tous les sérums ont été testés au Cerva. Les tests sérologiques utilisés ont été les suivants : le test au rose bengale (TRB), l'Elisa indirect (iElisa), le test de fixation du complément (TFC) et l'agglutination lente de Wright avec Edta (SAW-Edta) en microméthode. Les tests sérologiques pour la brucellose sont officiellement accrédités (normes ISO 17 025) par le Cerva d'Uccle.

Le TRB a été réalisé suivant la méthode décrite par Alton et coll. (2). L'iElisa l'a été suivant la méthode développée par Limet et coll. (14) en utilisant le biotype 1 de Brucella abortus (Weybridge 99) comme antigène. Le TFC a été réalisé sur des microplaques suivant la méthode préconisée dans le manuel de l'OIE (19). Enfin, la SAW-Edta a été effectuée d'après Garin et coll. (11).

\section{Analyse statistique}

Compte tenu de la distribution binaire des données de séroprévalence, une régression logistique a été réalisée à l'aide de Stata pour la comparaison entre types d'élevage (23). Une analyse bayésienne

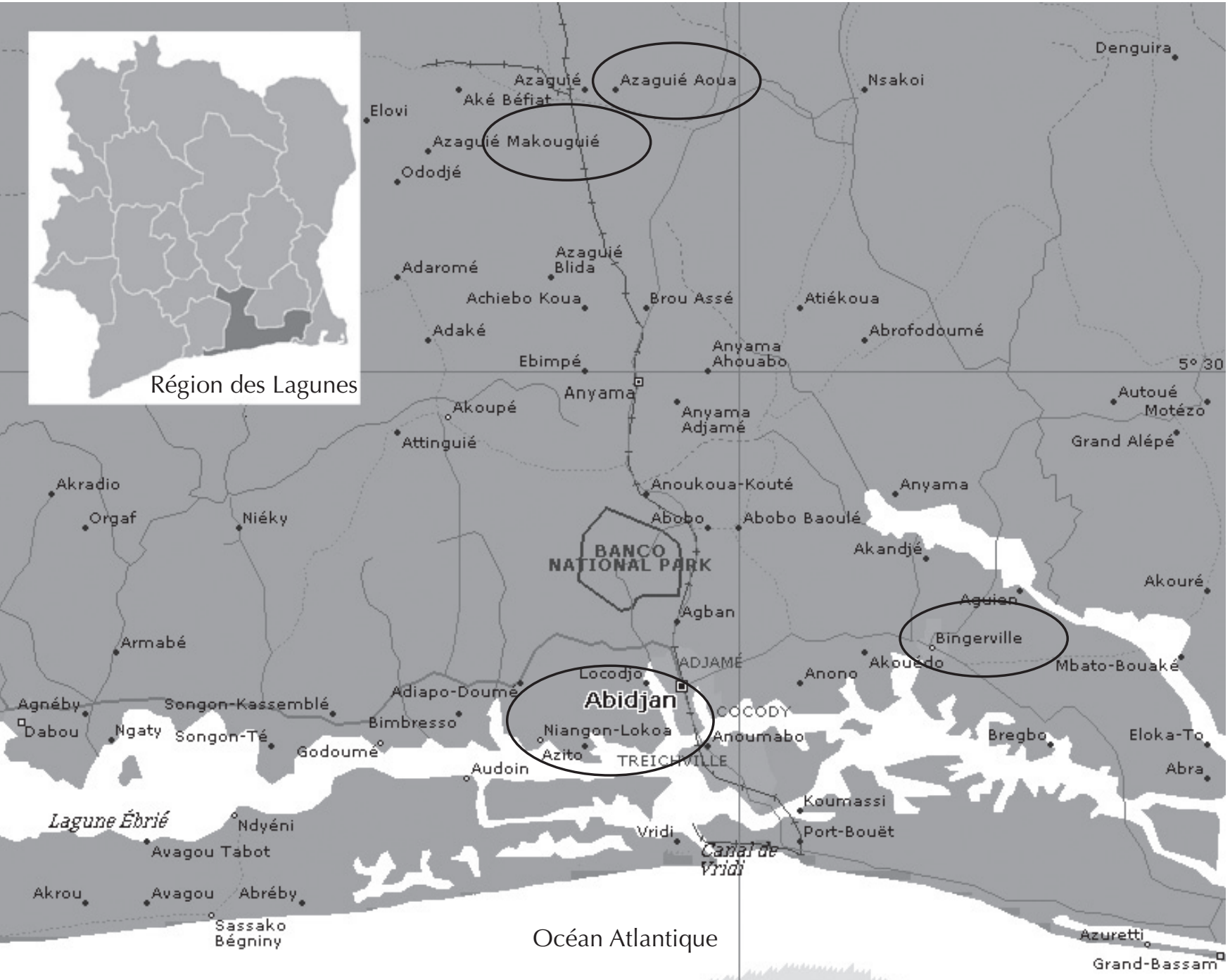

Figure 1 : zone d'étude avec les localités d'Abidjan, de Bingerville, d'Azaguié et de Makouguié (Côte d'Ivoire). 
a permis de combiner les informations préalables sur les sensibilités et les spécificités des tests sérologiques utilisés (appelées informations a priori) avec les résultats des analyses réalisées dans cette étude. La combinaison des informations a priori et de la vraisemblance des données (étant donné l'information a priori) aboutit à une distribution de probabilité a posteriori. Elle a été réalisée avec WinBUGS14 pour l'estimation de la prévalence de la brucellose et des caractéristiques des quatre tests sérologiques utilisés (sensibilité et spécificité). Les opinions d'experts ayant servi à la modélisation ont été extraits de Nielsen (18). Le résumé a été repris au tableau I.

\section{RESULTATS}

\section{Séroprévalence}

Les résultats de la séroprévalence sont résumés dans le tableau II. La régression logistique a montré qu'il n'y avait pas de différence significative statistiquement pour la séroprévalence entre les fermes suivies par le PLS et les élevages traditionnels au seuil de 5 p. 100.

La concordance entre les résultats des différents tests sérologiques utilisés est illustrée dans le tableau III.

\section{Tableau}

Sensibilité et spécificité des différents tests de diagnostic de la brucellose bovine et isotypes détectés

\begin{tabular}{|c|c|c|c|c|c|c|}
\hline \multirow[t]{2}{*}{ Tests } & \multirow{2}{*}{$\begin{array}{c}\text { Sensibilité } \\
(\%)\end{array}$} & \multirow{2}{*}{$\begin{array}{c}\text { Spécificité } \\
(\%)\end{array}$} & \multicolumn{4}{|c|}{ Anticorps détectés } \\
\hline & & & $\operatorname{Ig} M$ & $\operatorname{IgA}$ & $\operatorname{lgG}_{1}$ & $\lg G_{2}$ \\
\hline SAW-Edta & $29,1-100$ & $99,2-100$ & + & + & - & + \\
\hline TRB & $21-98,3$ & $68,8-100$ & + & - & + & + \\
\hline TFC & $23-97,1$ & $30,6-100$ & + & - & + & - \\
\hline Elisa & $92,5-100$ & $90,6-100$ & - & - & + & + \\
\hline
\end{tabular}

Source : Nielsen, 2002 (pour la sensibilité et la spécificité)

SAW-Edta : agglutination lente de Wright avec Edta ; TRB : test au rose bengale ; TFC : test de fixation du complément

\section{Tableau II}

Séroprévalence de la brucellose dans les différents types d'élevage en Côte d'Ivoire en utilisant différents tests sérologiques

\begin{tabular}{|c|c|c|c|c|c|}
\hline & \multirow[t]{2}{*}{$\begin{array}{l}\text { Nb. d'animaux } \\
\text { testés }\end{array}$} & \multicolumn{4}{|c|}{$\begin{array}{l}\text { Nb. d'examens positifs } \\
\text { et proportion (\%) }\end{array}$} \\
\hline & & SAW-Edta & TFC & TRB & Elisa \\
\hline $\begin{array}{l}\text { Fermes } \\
\text { laitières }\end{array}$ & 244 & $7(2,9)$ & $3(1,2)$ & $4(1,6)$ & $5(2)$ \\
\hline $\begin{array}{l}\text { Elevages } \\
\text { traditionnels }\end{array}$ & 137 & $5(3,6)$ & $3(2,2)$ & $4(2,9)$ & $4(2,9)$ \\
\hline Total & 381 & $12(3,1)$ & $6(1,6)$ & $8(2,1)$ & $9(2,4)$ \\
\hline
\end{tabular}

SAW-Edta : agglutination lente de Wright avec Edta ; TFC : test de fixation du complément ; TRB : test au rose bengale
Sur les 381 animaux testés, la majorité (362) étaient négatifs pour les quatre tests utilisés. Seuls cinq échantillons ont été positifs pour les quatre tests simultanément.

\section{Analyse bayésienne}

Ces résultats ont été utilisés pour la modélisation bayésienne (tableau IV).

Les valeurs intrinsèques des tests utilisés sont résumées dans le tableau V. Les premières caractéristiques concernent les fermes laitières et les deuxièmes les élevages traditionnels. Elles représentent les distributions postérieures qui résultent de la combinaison des opinions d'experts et de la vraisemblance des données.

\section{Tableau III}

Résultats bruts globaux des différents tests sérologiques utilisés pour tous les animaux provenant des élevages étudiés $(n=381)$

\begin{tabular}{ccccc} 
SAW-Edta & TFC & TRB & Elisa & Nb. bovins \\
\hline 0 & 0 & 0 & 0 & 362 \\
0 & 0 & 0 & 1 & 4 \\
0 & 0 & 1 & 0 & 2 \\
0 & 0 & 1 & 1 & 0 \\
0 & 1 & 0 & 0 & 1 \\
0 & 1 & 0 & 1 & 0 \\
0 & 1 & 1 & 0 & 0 \\
0 & 1 & 1 & 1 & 0 \\
1 & 0 & 0 & 0 & 6 \\
1 & 0 & 0 & 1 & 0 \\
1 & 0 & 1 & 0 & 1 \\
1 & 0 & 1 & 1 & 0 \\
1 & 1 & 0 & 0 & 0 \\
1 & 1 & 0 & 1 & 0 \\
1 & 1 & 1 & 0 & 0 \\
1 & 1 & 1 & 1 & 5
\end{tabular}

SAW-Edta : agglutination lente de Wright avec Edta ; TFC : test de fixation du complément ; TRB : test au rose bengale

0 : résultat négatif ; 1 : résultat positif

\section{Tableau IV}

Prévalences estimées de la brucellose et intervalles de crédibilité à partir de l'analyse bayésienne

\begin{tabular}{lccc} 
Type d'élevage & $\mathbf{n}$ & $\begin{array}{c}\text { Prévalence * } \\
(\%)\end{array}$ & $\begin{array}{c}\text { Intervalle de } \\
\text { crédibilité à 95 \% }\end{array}$ \\
\hline $\begin{array}{l}\text { Fermes } \\
\text { (application } \\
\text { et privées) }\end{array}$ & 244 & 3,573 & {$[1,164-7,113]$} \\
$\begin{array}{l}\text { Elevages } \\
\text { traditionnels }\end{array}$ & 137 & 4,291 & {$[1,324-8,755]$}
\end{tabular}

* A partir de la séropositivité dans les quatre tests utilisés 


\section{Tableau V}

Valeurs intrinsèques après analyse bayésienne des tests utilisés et intervalles de crédibilité à $95 \%$, au niveau (1) des fermes et (2) des élevages traditionnels

\begin{tabular}{lcccc} 
Test et valeur & SAW-Edta & TFC & TRB & Elisa \\
\hline 1) Sensibilité & 75,65 & 42,07 & 73,23 & 96,33 \\
& {$[37,71-98,85]$} & {$[23,78-75,42]$} & {$[30,52-97,44]$} & {$[92,70-99,83]$} \\
(2) Sensibilité & 81,84 & 60,28 & 78,12 & 96,39 \\
& {$[42,62-99,42]$} & {$[27,69-91,89]$} & {$[39,25-97,62]$} & {$[92,72-99,84]$} \\
(1) Spécificité & 99,62 & 99,31 & 98,89 & 98,44 \\
& {$[99,22-99,98]$} & {$[97,88-99,97]$} & {$[97,13-99,91]$} & {$[96,41-99,73]$} \\
(2) Spécificité & 99,61 & 99,22 & 99,25 & 98,61 \\
& {$[99,22-99,98]$} & {$[97,10-99,98]$} & {$[97,27-99,98]$} & {$[96,00-99,89]$}
\end{tabular}

SAW-Edta : agglutination lente de Wright avec Edta ; TFC : test de fixation du complément ; TRB : test au rose bengale

\section{DISCUSSION}

La prévalence de la brucellose bovine à partir de l'analyse bayésienne a été de 3,6 p. 100 et de 4,3 p. 100 respectivement dans les élevages laitiers et les troupeaux traditionnels. Ces chiffres étaient plus fiables que les chiffres obtenus dans les différents tests sérologiques. Une combinaison de plusieurs tests sérologiques donne une meilleure image de la situation, en l'absence de méthode de choix, tout en tenant compte du fait que des réactions croisées peuvent se manifester avec certaines bactéries comme Yersinia enterocolitica O:9, Xanthomonas maltophilia ou Salmonella urbana (22).

Les taux trouvés étaient faibles par rapport aux observations faites dans d'autres pays et régions d'Afrique subsaharienne, comme en Ouganda (9). En revanche, ils étaient très proches de la prévalence de 2,6 p. 100 trouvée dans la zone périurbaine d'Abéché (Tchad) (5). L'absence de différence entre les fermes laitières et les troupeaux traditionnels pourrait s'expliquer par la faible prévalence et une bonne gestion des troupeaux traditionnels dont les propriétaires étaient des citadins nantis.

L'analyse bayésienne, qui a permis aussi d'estimer la sensibilité et la spécificité des différents tests utilisés (tableau V), a montré que le test Elisa donnait la meilleure combinaison de sensibilité et de spécificité, ce qui est conforme aux observations de Nielsen (18), et Saegerman et coll. (22). En outre, ce test a permis de détecter des animaux porteurs latents (animaux classés négatifs par les tests classiques ; 22). Bien que la sensibilité du test au rose bengale n'ait pas été très élevée, ce test est reconnu pour sa simplicité de réalisation, surtout en milieu tropical, et son faible coût $(1,6)$. L'Elisa est utilisable dans les zones indemnes ou à prévalence faible comme en zone forestière de la Côte d'Ivoire (10). Les estimations ont également été le résultat de la prise en compte des opinions a priori, et Nielsen et coll. ont déjà recommandé ce test comme étant le plus sensible et le plus spécifique.

\section{CONCLUSION}

On peut conclure que la séroprévalence de la brucellose chez les animaux échantillonnés dans les élevages aux alentours d'Abidjan est très faible. Afin de confirmer que les quelques cas séropositifs sont de vrais cas de brucellose, il faudrait tester ces animaux en utilisant la technique de l'intradermoréaction à base de brucellergène - considérée comme le test le plus spécifique disponible actuellement malgré un manque de sensibilité - et en essayant d'isoler la souche de Brucella en question.

\section{Remerciements}

Les auteurs remercient M. Gragnon Biégo et l'équipe du terrain du Projet laitier Sud, principalement M. Baro, pour la collecte des échantillons sur le terrain, ainsi que Mmes C. de Smedt, M. Marin et S. Malbrecq du Cerva pour leur collaboration dans les analyses sérologiques. La Coopération technique belge (CTB), la direction générale de la Coopération au développement (Dgcd) et le Cerva sont remerciés pour leur appui financier.

\section{BIBLIOGRAPHIE}

1. AKAKPO A.J., 1987. Brucelloses animales en Afrique tropicale. Particularités épidémiologique, clinique et bactériologique. Revue Elev. Méd. vét. Pays trop., 40 : 307-320.

2. ALTON G.G., JONES L.M., ANGUS R.D., VERGER J.M., 1988. Techniques for the brucellosis laboratory. Paris, France, INRA, $190 \mathrm{p}$.

3. ANGBA A., TRAORE A., FRITZ P., 1987. Situation de la brucellose animale en Côte d'Ivoire. Revue Elev. Méd. vét. Pays trop., 40 : 325-329.

4. BANDARA A.B., MAHIPALA M.B., 2002. Incidence of brucellosis in Sri Lanka: an overview. Vet. Microbiol., 90: 197-207.

5. DELAFOSSE A., GOUTARD F., THEBAUD E., 2002. Epidémiologie de la tuberculose et de la brucellose des bovins en zone périurbaine d'Abéché, Tchad. Revue Elev. Méd. vét. Pays trop., 55 : 5-13.

6. DOMENECH J., LUCET P., GRILLET C., 1980. La brucellose bovine en Afrique centrale. I. Méthodes d'enquêtes utilisables en milieu tropical. Revue Elev. Méd. vét. Pays trop., 33 : 271-276.

7. DOMENECH J., LUCET P., VALLAT B., STEWART C., BONNET J.B., HENTIC A., 1982. La brucellose bovine en Afrique centrale. III. Résultats statistiques des enquêtes menées au Tchad et au Cameroun. Revue Elev. Méd. vét. Pays trop., 35 : 15-22.

8. FAO, 2003. Guidelines for coordinated human and animal brucellosis surveillance. Rome, Italy, FAO, 45 p. (Animal Production and Health Paper No 156)

9. FAYE B., CASTEL V., LESNOFF M., RUTABINDA D., DHALWA J., 2005. Tuberculosis and brucellosis prevalence survey on dairy cattle in Mbarara milk basin (Uganda). Prev. vet. Med., 67: 267-281.

10. GALL D., NIELSEN K., 2004. Serological diagnosis of bovine brucellosis: a review of test performance and cost comparison. Revue sci. tech. Off. int. Epizoot., 23: 989-1002.

11. GARIN B., TRAP D., GAUMONT R., 1985. Assessment of the EDTA seroagglutination test for the diagnosis of bovine brucellosis. Vet. Rec., 117: 444-445

12. GODFROID J., BISHOP G.C., BOSMAN P.P., HERR S., 2004. Bovine brucellosis. In: Coetzer J.A.W., Tustin R.C. Eds, Infectious diseases of livestock. Cape Town, South Africa, Oxford University Press, p. 15101527. 
13. LEFEVRE P.C., 1991. Atlas des maladies infectieuses des ruminants. Maisons-Alfort, France, Cirad-lemvt, 95 p.

14. LIMET J.N., KERKHOFS P., WIJFFELS R., DEKEYSER P., 1988. Le diagnostic sérologique de la brucellose bovine par Elisa. Ann. Méd. vét., 132 : 565-575.

15. MCDERMOTT J.J., ARIMI S.M., 2002. Brucellosis in sub-Saharan Africa: epidemiology, control and impact. Vet. Microbiol., 90: 111-134.

16. MORENO E., 2002. Brucellosis in Central America. Vet. Microbiol., 90: 31-38.

17. MUSTAFA A.A., NICOLETTI P., 1995. FAO, WHO, OIE guidelines for a regional brucellosis control programme for the Middle East. http://www.fao.org/ag/AGA/AGAH/ID/GUIDE-EN.htm

18. NIELSEN K., 2002. Diagnosis of brucellosis by serology. Vet. Microbiol., 90: 447-459.

19. OIE, 2000. Bovine brucellosis. In: Manual of standards diagnostic tests and vaccines. Paris, France, OIE, p. 328-345.
20. PILO-MORON E., PIERRE F., KOUAME J.B., 1979. Brucellose bovine en Côte d'ivoire. Epidémiologie. Revue Elev. Méd. vét. Pays trop., 32 325-333.

21. RICHEY E.J., DIX HARREL C., 1997. Brucella abortus disease (brucellosis) in beef cattle. Gainesville, FL, USA, Florida Cooperative Extension Service, Institute of Food and Agricultural Sciences, 6 p.

22. SAEGERMAN C., DE WAELE L., GILSON D., GODFROID J., THIANGE P., MICHEL P., LIMBOURG B., VO T.K.O., LIMET J., LETESSON J.J., BERKVENS D., 2004. Evaluation of three serum i-ELISAs using monoclonal antibodies and protein $G$ as peroxidase conjugate for the diagnosis of bovine brucellosis. Vet. Microbiol., 100: 91-105.

23. STATA CORP., 2001. Stata statistical software, Vers. 7.0. College Station, TX, USA, Stata Corporation

24. WILLIAMS D.J.L., AKANMORI B.D., WASTLING J.M., 2000. Tropical zoonosis in West Africa. Acta trop., 76: 1-83.

Reçu le 04.11.2005, accepté le 27.03.2006

\section{Summary}

Thys E., Yahaya M.A., Walravens K., Baudoux C., Bagayoko I., Berkvens D., Geerts S. Study of the Prevalence of Bovine Brucellosis in the Forest Zone of Cote d'Ivoire

A serological survey was carried out in the suburban forest zone of Abidjan from July to September 2004 to estimate the prevalence of brucellosis in dairy cattle. Three hundred and eighty one (381) serums were collected from a total of 927 animals, of which 506 originated from the dairy farms supervised by the South Dairy Project (demonstration farm and private farms), and 421 from neighboring traditional herds. Four serological tests were performed: the rose bengal test, indirect ELISA, the complement fixation test, and the slow agglutination test of Wright with EDTA (micromethod). On the basis of serological and bayesian analyses, seroprevalence rates of about 3.6 and $4.3 \%$ were found in the dairy farms and traditional herds, respectively. No significant difference was found between the farms and traditional herds $(p<0.05)$.

Keywords: Cattle - Brucella - Brucellosis - Morbidity - Immunological technique - Suburban area - Forest - Cote d'Ivoire.

\section{Resumen}

Thys E., Yahaya M.A., Walravens K., Baudoux C., Bagayoko I., Berkvens D., Geerts S. Estudio de la prevalencia de la brucelosis bovina en zonas forestales de la Costa de Marfil

Se llevó a cabo una encuesta serológica en la zona forestal peri urbana de Abidján, entre julio y septiembre 2004, con el fin de evaluar la prevalencia de la brucelosis en el hato lechero bovino. Se recolectaron trescientos ochenta y un (381) sueros, sobre un total de 927 animales, de los cuales 506 provenían de fincas lecheras seguidas por el Proyecto lechero del Sur (fincas de aplicación y privadas) y 421 de las crías tradicionales de los alrededores. Se aplicaron cuatro tests serológicos: el test del rosa de bengala, ELISA indirecto, test de fijación de complemento y aglutinación lenta de Wright con EDTA (micro método). Sobre la base de los análisis serológicos y bayesiana, se obtuvieron seroprevalencias del orden de 3,6 y de $4,3 \%$ respectivamente en las fincas lecheras y en las crías tradicionales. No hubo una diferencia significativa entre las fincas lecheras y las crías tradicionales $(p<0,05)$.

Palabras clave: Ganado bovino - Brucella - Brucelosis Morbosidad - Técnica inmunológica - Zona periurbana Bosque - Cote d'Ivoire. 